\title{
Die diskreten Gruppen euklidischer Bewegungen in der Ebene
}

\author{
Walter Wolking \\ Walter Wolking, geboren 1941, studierte Mathematik und Physik an der Universität \\ Münster. Er promovierte 1978 an der Ruhruniversität in Bochum, versah anschlies- \\ send während mehrerer Jahre Lehraufträge an der Universität in Osnabrück und ist \\ heute als Gymnasiallehrer tätig. Sein Hauptinteresse gehört den Kleinschen Gruppen, \\ insbesondere dem Hausdorff-Mass und der Hausdorff-Dimension ihrer Grenzpunkt- \\ menge. Entspannung von der mathematischen Arbeit sucht er am liebsten in der \\ freien Natur.
}

\section{Einleitung}

Die Drehungen und Parallelverschiebungen (Translationen) in der Ebene sind genau die Transformationen, die man durch zwei aufeinander folgende Geradenspiegelungen erhält. Schaltet man zwei derartige Transformationen hintereinander, so ist das Ergebnis wiederum eine Drehung oder Translation. Die geraden Mehrfachspiegelungen bilden demnach die Gruppe $M$ aller Drehungen und Translationen in der Gruppe $\bar{M}$ aller Mehrfachspiegelungen (euklidische Bewegungen).

$M$ ist wiederum eine Untergruppe der Gruppe $M_{L}$ aller geraden Mehrfachspiegelungen an Geraden und Kreisen (Gruppe der gebrochen linearen Transformationen $z \rightarrow \frac{a z+b}{c z+d}$ ). Die 'Kleinschen Gruppen' aus $M_{L}$ sind durch die im folgenden erläuterte Eigenschaft (*) charakterisiert. Sie spielen eine große Rolle bei der Untersuchung und Beschreibung Riemannscher Flächen (Flächen mit einer komplexen Struktur). Jede derartige Fläche erhält man aus einem im weiteren näher beschriebenen Fundamentalgebiet dadurch, daß

Es ist eine Mannigfaltigkeit und in derselben eine Transformationsgruppe gegeben; man solle die der Mannigfaltigkeit angehörigen Gebilde hinsichtlich solcher Eigenschaften untersuchen, die durch die Transformationen der Gruppe nicht geändert werden. Gemäss dem Erlanger Programm von Felix Klein (1872), aus dem dieses Zitat stammt, gehört die Gruppe der euklidischen Bewegungen der Ebene zur ebenen Kongruenzgeometrie. Algebraische Aussagen über diese Gruppe entsprechen kongruenzgeometrischen Aussagen in der Ebene. Im vorliegenden Beitrag stellt sich Walter Wolking die Aufgabe, alle diskreten Untergruppen zu beschreiben; diese entsprechen Parkettierungen der Ebene durch kongruente Bereiche. ust 
man äquivalente Punkte des Randes identifiziert. Kennt man die Untergruppen aus $M_{L}$ mit der Eigenschaft $(*)$, so hat man einen Überblick über alle Riemannschen Flächen. Im folgenden werden u.a. diejenigen diskreten (Kleinschen) Gruppen $G$ bestimmt, bei denen sich die Bilder eines Fundamentalgebietes genau in einem Punkt (hier Grenzpunkt $\infty)$ häufen. Die Anzahl der Grenzpunkte einer Kleinschen Gruppe ist 0 oder 1 oder 2 oder unendlich. Ist $U$ einfach periodisch, so entspricht im Falle $G=U$ der zugehörigen Riemannschen Fläche topologisch eine Sphäre, der zwei Punkte entnommen sind (zweifach punktierte Sphäre), im Falle $G \neq U$ eine einfach punktierte Sphäre mit zwei Verzweigungspunkten (entsprechen den Drehzentren). Ist $G$ doppelt periodisch, so ist die Riemannsche Fläche ein Torus, in den übrigen Fällen eine Sphäre mit maximal vier Verzweigungspunkten. Unendlich viele Exemplare eines Fundamentalgebietes überdecken die gesamte Ebene. Es werden hier also u.a. mit elementaren geometrischen Voraussetzungen, ohne Zuhilfenahme der komplexen Analysis, sämtliche Riemannschen Flächen bestimmt, die durch die komplexe Ebene (i.a. verzweigt) regulär überlagert werden.

Benutzt man zusätzlich die Spiegelungen an Kreisen, so kann man unter einfachen Voraussetzungen auch die diskreten Gruppen ohne Grenzpunkt bzw. mit genau zwei Grenzpunkten bestimmen. Zudem läßt sich mit Hilfe der vorliegenden Untersuchungen ein Überblick über die entsprechenden diskreten Gruppen im Raum $\mathbb{R}^{3}$ und damit über die zugehörigen dreidimensionalen Mannigfaltigkeiten gewinnen; die Verhältnisse sind dort jedoch i.a. komplizierter und schwieriger darzustellen.

Jede Transformation aus $M$ läßt sich durch maximal drei Geradenspiegelungen darstellen. Jede Dreifachspiegelung kann durch eine einfache Spiegelung mit eventueller anschließender Translation parallel zur Spiegelachse beschrieben werden.

Eine Spiegelung mit anschließender Translation parallel zur Spiegelachse bezeichnet man als Gleitspiegelung.

Zwei verschiedene Punkte der Ebene sind äquivalent unter $M$, d.h. es gibt eine Transformation aus $M$, die einen der Punkte auf den anderen abbildet. Insbesondere enthält jede noch so kleine Kreisumgebung eines beliebigen Punktes äquivalente Punkte unter $M$. Die im letzten Satz gekennzeichnete Eigenschaft trifft auf zahlreiche Untergruppen von $M$ zu. So ist jede Drehung $j$ mit dem Drehwinkel $a \pi, a$ irrational, wegen $n a \neq k$ für beliebige $k, n \in \mathbb{Z}$, von unendlicher Ordnung $(\operatorname{ord}(j)=\infty)$, d.h. die von $j$ erzeugte Untergruppe enthält unendlich viele Elemente und damit Drehungen mit beliebig kleinem Drehwinkel.

Untergruppen mit der obigen Eigenschaft sind i.a. wenig interessant. In dieser Arbeit sollen diejenigen Untergruppen $W$ aus $\bar{M}$ bestimmt werden, auf die die obige Eigenschaft nicht zutrifft. Gesucht sind also sämtliche Untergruppen $W \subset \bar{M}$ mit folgender Eigenschaft:

$(*)$ Es gibt mindestens einen Punkt $z_{0}$ der Ebene und dazu eine offene Kreisumgebung $U_{z_{0}} ; U_{z_{0}}=\left\{z|| z-z_{0} \mid<r\right\}$; in der keine äquivalenten Punkte unter $W$ liegen.

Die Kreisscheiben $S\left(U_{z_{0}}\right)$ mit $S \in W$ überschneiden sich also nicht.

Die Eigenschaft $(*)$ charakterisiert die diskreten Untergruppen. Die Bezeichnung läßt sich für Untergruppen aus $M$ wie folgt motivieren: 
Jede Drehung kann man durch eine Drehung um den Nullpunkt (mit gleichem Drehwinkel) und anschließende Translation darstellen - zum Beweise wähle man die Verbindungsgerade vom Nullpunkt zum Drehzentrum als eine Spiegelachse. Jedes Element aus $M$ ist somit durch ein Tripel $\left(x_{1}\left|x_{2}\right| x_{3}\right)$, also einen Punkt aus $\mathbb{R}^{3}$, gekennzeichnet, wobei $x_{1}$ den Drehwinkel und $\left(x_{2} \mid x_{3}\right)$ den Translationsvektor angibt; für reine Translationen ist $x_{1}=0+n 2 \pi . x_{2}, x_{3}$ sind eindeutig, $x_{1}$ dagegen nur bis auf ganzzahlige Vielfache von $2 \pi$ bestimmt, d.h. zwei Punkte aus $\mathbb{R}^{3}$ bestimmen genau dann dasselbe Element aus $M$, wenn sie in der 2. und 3. Koordinate übereinstimmen und die Differenz der 1. Koordinaten ein ganzzahliges Vielfaches von $2 \pi$ ist. Die zu $M$ gehörende Punktmenge $M_{p}$ ist der gesamte Raum $\mathbb{R}^{3}$. Hat dagegen $G \subset M$ die Eigenschaft (*), so gibt es in $G$ keine Transformationen, die der identischen Abbildung beliebig nahekommen, d.h., es gibt eine Umgebung des Nullpunktes in $\mathbb{R}^{3}$, in der kein Punkt der Menge $G_{p}$ liegt. Aufgrund des Gruppencharakters von $G$ liegen die Punkte von $G_{p}$ isoliert in $\mathbb{R}^{3}$; es handelt sich um eine diskrete Punktmenge. Nach diesen Vorüberlegungen lassen sich folgende Äquivalenzen leicht beweisen:

$G \subset M$ hat die Eigenschaft $(*) \Leftrightarrow$ Es gibt eine Umgebung des Nullpunktes in $\mathbb{R}^{3}$, in der kein Punkt aus $G_{p}$ liegt. $\Leftrightarrow$ Es gibt keine Folge $S_{n}\left(S_{n} \neq i d\right)$ von Transformationen aus $G$ mit $S_{n}(z) \rightarrow z$ für alle Punkte $z$ der Ebene.

Zum Nachweis der Eigenschaft $(*)$ bestimmen wir im folgenden Fundamentalgebiete (Fundamentaldreiecke, Fundamentalrauten).

Ein Fundamentalgebiet $F$ einer Gruppe $W \subset \bar{M}$ ist die abgeschlossene Punktmenge einer Fläche, die von Geraden oder Halbgeraden oder Strecken berandet wird (einschließlich der Randpunkte), mit folgender Eigenschaft:

Die Bildflächen $S(F)$ überdecken die ganze Ebene, überlappen sich jedoch nicht. Zwei verschiedene Bildfächen haben somit höchstens gemeinsame Randpunkte.

Um alle diskreten Gruppen $\bar{G} \subset \bar{M}$ zu erfassen, bestimmen wir zunächst sämtliche diskreten Gruppen $G \subset M$ und untersuchen unmittelbar im Anschluß daran alle Möglichkeiten der Erweiterung von $G$ zu einer diskreten Gruppe $\bar{G}$ derart, daß die Untergruppe der geraden Mehrfachspiegelungen aus $\bar{G}$ genau $G$ ist. $\bar{G}$ ist im allgemeinen durch $G$ nicht eindeutig bestimmt. Da die geraden Mehrfachspiegelungen einer diskreten Gruppe $\bar{G} \subset \bar{M}$ eine diskrete Gruppe $G \subset M$ bilden, haben wir damit einen vollständigen Überblick über alle diskreten Gruppen euklidischer Bewegungen.

Zur Bestimmung sämtlicher diskreten Gruppen $G \subset M$ machen wir eine grobe Fallunterscheidung über die Untergruppen $U(U \subset G)$ aller Translationen.

Die Untergruppe $U$ heißt einfach periodisch genau dann, wenn sie von genau einem Element erzeugt wird.

Ist dies nicht der Fall, so gibt es zwei Translationen aus $U$, deren Translationsvektoren linear unabhängig sind. $U$ wird dann von genau zwei Elementen erzeugt; wir nennen $U$ in diesem Fall doppelt periodisch.

Da eine Hintereinanderschaltung zweier ungerader Mehrfachspiegelungen (Gleitspiegelungen) aus $\bar{G}$ ein Element aus $G$ ist, erzeugt jede Gleitspiegelung aus $\bar{G}$ zusammen mit Erzeugenden der Gruppe $G$ die ganze Gruppe $\bar{G}$. Diese zusätzliche Erzeugende wollen wir im folgenden grundsätzlich mit $e$, die zugehörige Spiegelachse mit $a$ bezeichnen. 
Besteht $G$ nur aus der identischen Abbildung id und ist $e=s p \circ t=t \circ s p$ in $\bar{G}$, so gilt $e^{2}=t \circ s p \circ s p \circ t=t^{2} \in G$. Es muß dann $t=i d$ sein; $e$ ist eine einfache Spiegelung. Die Gruppen $\bar{G}$ mit $G=\{i d\}$ werden durch einfache Spiegelungen erzeugt.

In den folgenden Büchern findet der Leser weitere Informationen über dieses Thema [1], [2], [3], [4].

\section{Die diskreten Gruppen von Drehungen}

Wir beweisen zunächst:

(1) Enthält $G$ ausschließlich Drehungen, so ist $G$ zyklisch mit endlich vielen Elementen.

Dazu zeigen wir:

(2) Enthält $G$ zwei Drehungen $j_{1}, j_{2}$ mit verschiedenen Fixpunkten, so gibt es in $G$ eine Translation.

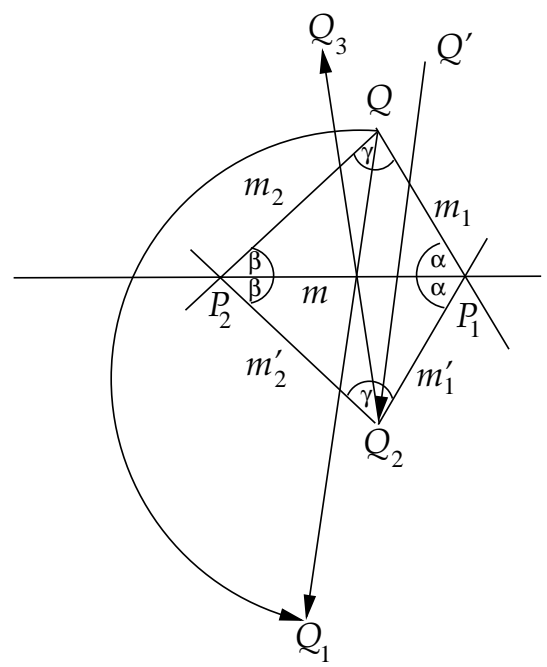

Abb. 1

Die Beweisschritte zu (2) lassen sich anhand der Abb. 1 anschaulich interpretieren. Wir können annehmen, daß die Drehwinkel $\alpha, \beta$ von $j_{1}, j_{2}$ (mit den Fixpunkten $P_{1}, P_{2}$ ) positiv sind.

Dann gilt

$$
\begin{aligned}
j_{1}= & s p(m) \circ s p\left(m_{1}\right), \quad(s p(u)-\text { Spiegelung an der Achse } u) \\
j_{2}= & s p\left(m_{2}\right) \circ s p(m), \\
j_{2} \circ j_{1}= & s p\left(m_{2}\right) \circ s p(m) \circ s p(m) \circ s p\left(m_{1}\right)=s p\left(m_{2}\right) \circ s p\left(m_{1}\right) \\
& (\text { Drehung um } Q \text { mit dem Drehwinkel }-2 \gamma) \\
j_{1}^{-1}= & s p(m) \circ s p\left(m_{1}^{\prime}\right), \\
j_{2}^{-1}= & s p\left(m_{2}^{\prime}\right) \circ s p(m), \\
j_{2}^{-1} \circ j_{1}^{-1}= & s p\left(m_{2}^{\prime}\right) \circ s p\left(m_{1}^{\prime}\right)
\end{aligned}
$$

(Drehung um $Q_{2}$ mit dem Drehwinkel $2 \gamma$ ) 
Die Abbildung $t_{1}=j_{2}^{-1} \circ j_{1}^{-1} \circ j_{2} \circ j_{1}$ bildet $Q$ auf $Q_{1}$ und $\left(j_{2} \circ j_{1}\right)^{-1}\left(Q_{2}\right)=Q^{\prime}$ auf $Q_{2}$ ab.

Aufgrund der Symmetrie sind die Strecken $Q Q_{1}$ und $Q^{\prime} Q_{2}$ gleichlang. Wegen $t_{1}(Q)=$ $Q_{1}, t_{1}\left(Q^{\prime}\right)=Q_{2}$ und $t_{1}(\infty)=\infty$ ist $t_{1}$ eine Translation. Die obigen Überlegungen gehen von der Voraussetzung aus, $\mathrm{da} \beta m_{1}$ und $m_{2}$ sich schneiden. Verlaufen $m_{1}, m_{2}$ parallel, so ist $j_{2} \circ j_{1}=s p\left(m_{2}\right) \circ s p\left(m_{1}\right)$ eine Translation.

Damit ist (2) bewiesen.

Anhand der Symmetrie der Abbildung 1 sieht man weiterhin, daß $t_{2}=j_{2} \circ j_{1} \circ j_{2}^{-1} \circ j_{1}^{-1}$ eine Translation mit $t_{2}\left(Q_{2}\right)=Q_{3}$ ist. Ist $\gamma \neq 90^{\circ}$, so sind die Vektoren $\overrightarrow{Q_{Q_{1}}}$ und $\overrightarrow{Q_{2} Q_{3}}$ linear unabhängig; die von $j_{1}, j_{2}$ erzeugte Gruppe enthält dann eine doppelt periodische Untergruppe.

Aus (2) folgt, daß die Elemente der in (1) vorausgesetzten Gruppe $G$ einen gemeinsamen endlichen Fixpunkt haben. Da $G$ diskret sein soll, können die Drehwinkel der Elemente nicht beliebig klein werden, d.h. es gibt eine Drehung $j_{0}$ in $G$ mit minimalem (positivem) Drehwinkel. Jede andere Drehung in $G$ muß dann eine mehrfache Hintereinanderschaltung von $j_{0}$ sein, da andernfalls $j_{0}$ nicht minimal ist. Aus dem gleichen Grund muß ein Vielfaches des Drehwinkels von $j_{0}$ gleich $2 \pi$ sein.

Somit ist (1) vollständig bewiesen.

Die endlichen zyklischen Gruppen von Drehungen sind genau die diskreten Gruppen euklidischer Bewegungen, die ausschließlich aus Drehungen bestehen.

Jede Gleitspiegelung $e \in \bar{G}$ bildet den endlichen Fixpunkt $P$ von $G$ wieder auf einen Fixpunkt einer Transformation aus $G$ ab. Also kann $e$ nur eine Spiegelung sein, deren Achse $a$ durch $P$ verläuft.

Sind $a, m$ beliebige Achsen durch $P$ mit $j_{0}=s p(a) \circ s p(m)$, so erzeugen die Spiegelungen $s p(a), s p(m)$ eine Gruppe $\bar{G}$. Ein von $a, m$ gebildeter Sektor ist dann Fundamentalgebiet von $\bar{G}$.

\section{Die diskreten Gruppen von Translationen}

Wir setzen nun voraus, daß $G$ ausschließlich Translationen enthält. Unter den zugehörigen Perioden $z_{t}$ muß es dann eine Periode $z_{t_{0}}$ mit minimalem Betrag geben, da andernfalls eine Folge $t_{n} \in G$ existiert, die gegen die identische Abbildung strebt. $t_{0}$ erzeugt eine einfach periodische Untergruppe $U_{t_{0}}$ (zyklisch vom Rang 1) von $G$. Ist $U_{t_{0}} \neq G$, so gibt es eine Translation $t_{1}$ in $G-U_{t_{0}}$, deren Vektor $z_{t_{1}}$ von allen Translationsvektoren der zu $G-U_{t_{0}}$ gehörenden Elemente minimalen Betrag hat.

$z_{t_{1}}, z_{t_{0}}$ sind nicht kollinear. Sie bestimmen eine doppelt periodische Untergruppe $U_{t_{0} t_{1}} \subseteq$ $G$, die aus allen Translationen $t_{m, n}$ mit $t_{m, n}(z)=z+m z_{t_{0}}+n z_{t_{1}}$ besteht. $z_{t_{0}}, z_{t_{1}}$ sind nicht eindeutig. Sie können wegen $\left|z_{t_{1}}-z_{t_{0}}\right| \geq\left|z_{t_{1}}\right| \geq\left|z_{t_{0}}\right|$ so gewählt werden, daß $60^{\circ} \leq \Varangle\left(z_{t_{0}}, z_{t_{1}}\right)$ oder $\Varangle\left(z_{t_{1}}, z_{t_{0}}\right) \leq 90^{\circ}$ gilt.

Sämtliche zu den Elementen aus $U_{t_{0} t_{1}}$ gehörenden Perioden bestimmen in der Gaußschen Zahlenebene $E$ ein Gitter $G i$, dessen Punkte Bildpunkte der Eckpunkte des von $z_{t_{0}}, z_{t_{1}}$ aufgespannten abgeschlossenen Parallelogramms $P$ unter $U_{t_{0} t_{1}}$ sind. Man erkennt unschwer, daß in $P$ aufgrund der Wahl von $t_{0}, t_{1}$ keine weiteren Perioden liegen können und somit $U_{t_{0} t_{1}}=G$ gilt. Damit folgt: 
(3) Die diskreten Gruppen von Translationen sind die einfach periodischen (zyklisch vom Rang 1) und die doppelt periodischen Gruppen (vom Rang 2).

Zur Bestimmung der Gruppen $\bar{G}$ untersuchen wir im weiteren die Möglichkeiten für die zusätzlichen Erzeugenden $e$ und deren zugehörige Spiegelachsen $a$. Dazu beweisen wir zunächst einige Aussagen:

(4) Ist $e \in \bar{G}, e=s p(a) \circ t$ eine Gleitspiegelung mit $t \neq i d$, so gibt es eine Translation $t^{\prime} \in G$ mit $\left|z_{t}\right|=\frac{1}{2}\left|z_{t^{\prime}}\right|$.

Der Beweis folgt unmittelbar aus $e^{2}=t^{2}=t^{\prime} \in G$.

(5) Ist $e \in \bar{G}, e=s p(a) \circ t$ eine Gleitspiegelung und $t^{\prime}$ eine Translation aus $G$, so ist die Spiegelung von $z_{t^{\prime}}$ an $a$ wiederum der Translationsvektor einer Translation aus $G$.

Zum Beweise beachten wir, daß $e^{-1} \circ t^{\prime} \circ e=s p(a) \circ t^{-1} \circ t^{\prime} \circ t \circ s p(a)=s p(a) \circ t^{\prime} \circ s p(a)$ eine Translation ist, deren Translationsvektor man durch Spiegelung von $z_{t^{\prime}}$ an $a$ erhält.

(6) Sind $e_{1}=s p\left(a_{1}\right) \circ t_{1}$ und $e_{2}=s p\left(a_{2}\right) \circ t_{2}$ zwei Gleitspiegelungen mit nicht parallelen Achsen $a_{1}, a_{2}$, so ist $e_{2} \circ e_{1}$ eine Drehung.

Es gilt: $e_{2} \circ e_{1}=s p\left(a_{2}\right) \circ t_{2} \circ s p\left(a_{1}\right) \circ t_{1}=s p\left(a_{2}\right) \circ s p\left(a_{1}\right) \circ t^{\prime} \circ t_{1}=j^{\prime} \circ t=j$.

(7) Ist jede Transformation der von $e$ (Gleitspiegelung) und $G$ erzeugten Gruppe $G^{\prime}$ in der Form $g \circ e^{i}$ mit $g \in G, i \in\{0,1\}$, darstellbar, so gilt $G^{\prime}=\bar{G}$.

Jede gerade Mehrfachspiegelung aus $G^{\prime}$ hat die Darstellung $g \circ e^{0}\left(e^{0}=i d\right)$, ist also ein Element aus G. Die obige Darstellung ist eindeutig.

(8) Es seien $t, t^{\prime}$ Erzeugende einer doppelt periodischen Gruppe $G$ von Translationen mit den Translationsvektoren $z_{t}$ bzw. $z_{t^{\prime}}$. Wir können (eventuell durch Nachschaltung von $t^{m}$ mit geeignetem $m \in Z$ und durch Übergang von $t$ zu $t^{-1}$ ) davon ausgehen, daß die Komponente $p^{\prime}$ von $z_{t^{\prime}}$ parallel zu $z_{t}$ in Richtung von $z_{t}$ zeigt und vom Betrage her kleiner oder gleich $\frac{1}{2}\left|z_{t}\right|$ ist. Parallel zu $z_{t}$ verlaufende Geraden treten nur dann als Spiegelachsen $a$ von zusätzlichen Erzeugenden $e \in \bar{G}$ auf, wenn $p^{\prime}=0$ oder $p^{\prime}=\frac{1}{2} z_{t}$ gilt. Im Falle $p^{\prime}=0$ sind die Gruppen $\bar{G}$ durch $e=s p(a) \circ \tilde{t}$ mit $z_{\tilde{t}}=k \frac{1}{2} z_{t}, k \in\{0,1\}$, im Falle $p^{\prime}=\frac{1}{2} z_{t}$ durch $e=s p(a)$ bestimmt.

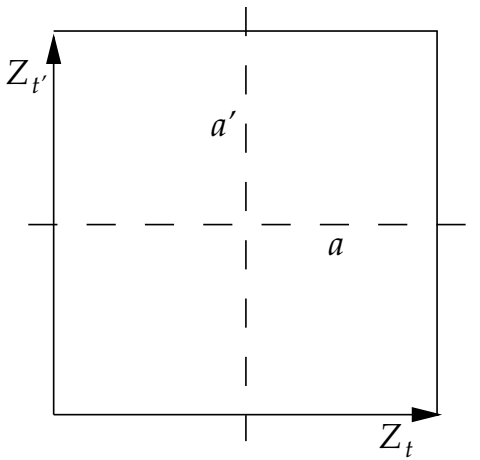

Abb. 2 
Nach (5) muß die Spiegelung $\overline{z_{t^{\prime}}}$ von $z_{t^{\prime}}$ an $a$ wiederum ein Translationsvektor einer Translation aus $G$ sein. Dann gilt $\overline{z_{t^{\prime}}}+z_{t^{\prime}}=0$ oder $\overline{z_{t^{\prime}}}+z_{t^{\prime}}=z_{t}$. Hieraus folgen die notwendigen Bedingungen $p^{\prime}=0$ bzw. $p^{\prime}=\frac{1}{2} z_{t}$. Ist $p^{\prime}=0$, so ist das von $z_{t}, z_{t^{\prime}}$ aufgespannte Fundamentalparallelogramm ein Rechteck. Wegen $e \circ t^{i}=s p(a) \circ \tilde{t} \circ t^{i}=$ $t^{i} \circ s p(a) \circ \tilde{t}=t^{i} \circ e, e \circ t^{\prime i}=t^{\prime-i} \circ e, e^{2}=t^{k} ; i=1,-1 ; k \in\{0,1\}$ folgt aus (7) und (4) die Behauptung, denn jede Gruppe $\bar{G}$ mit einer Gleitspiegelung $s p(a) \circ t^{*}$ enthält nach (4) eine der oben angegebenen Erzeugenden. Jede der durch $a$ bestimmten Hälften des Fundamentalrechtecks ist ein Fundamentalgebiet für $\bar{G}$.

Vertauscht man die Rollen von $t, t^{\prime}$, so erhält man die Möglichkeiten mit der Spiegelachse $a^{\prime}$ (s. Abb. 2).

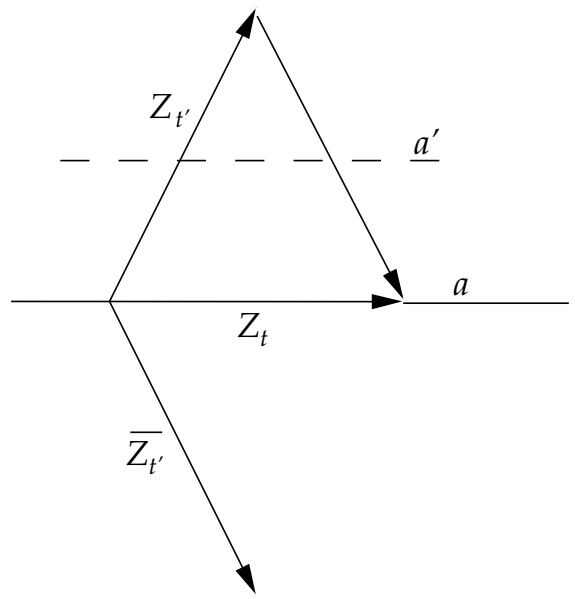

Abb. 3

Ist $p^{\prime}=\frac{1}{2} z_{t}$, so kann $e=s p(a) \circ \tilde{t}$ mit $z_{\tilde{t}}=\frac{1}{2} z_{t}$ durch $e \circ t^{\prime-1}=s p(a) \circ \tilde{t} \circ t^{\prime-1}=$ $s p(a) \circ t^{\prime \prime}=s p\left(a^{\prime}\right)$ ersetzt werden, mit $z_{t^{\prime \prime}} \perp a ; a^{\prime} \| a$.

Wegen $s p\left(a^{\prime}\right) \circ t^{i}=t^{i} \circ s p\left(a^{\prime}\right) ; s p\left(a^{\prime}\right) \circ t^{\prime i}=t^{i} \circ t^{\prime-i} \circ s p\left(a^{\prime}\right), i=1,-1$ (s. Abb. 3), sind nach (7) alle Möglichkeiten für $\bar{G}$ unter diesen Voraussetzungen (Spiegelachse $a$ parallel $\left.\mathrm{zu} z_{t}\right)$ durch $e=s p(a)$ bestimmt.

Die Translationsvektoren $z_{t^{\prime}}, \overline{z_{t^{\prime}}}$ spannen eine Raute auf; die zugehörigen Translationen sind Erzeugende von $G$. Ersetzt man $t$ durch die Translation mit dem Vektor $z_{t^{\prime}}-\overline{z_{t^{\prime}}}$, so erkennt man, daß durch $e=s p(a)$ alle Möglichkeiten mit $a \perp z_{t}$ für $\bar{G}$ erfaßt sind. Die Einfachspiegelungen an Geraden parallel zu den Diagonalen der Raute bestimmen unter den obigen Voraussetzungen $\left(a \| z_{t}, a \perp z_{t}\right.$ sämtliche Möglichkeiten für $\bar{G}$. Die durch die Diagonalen bestimmten Dreiecke der Raute sind dann jeweils Fundamentalgebiete von $\bar{G}$.

Es sei nun $G(=U)$ einfach periodisch mit der Erzeugenden $t_{0}$.

Nach (5) können als Spiegelachsen $a$ nur senkrecht und parallel zu $z_{t_{0}}$ verlaufende Geraden auftreten. Im ersten Fall kommt nach (4) nur $e=s p\left(a^{\prime}\right)$ in Frage (s. Abb. 4). Es gilt $s p\left(a^{\prime}\right) \circ t_{0}^{i}=t_{0}^{-i} \circ s p\left(a^{\prime}\right), i=1,-1$; also ist nach (7) $e=s p\left(a^{\prime}\right)$ eine zusätzliche Erzeugende. Der von $a^{\prime}, b$ berandete Streifen ist ein Fundamentalgebiet für $\bar{G}$. Wegen 
$s p\left(a^{\prime}\right) \circ t_{0}=s p(b)$ wird $\bar{G}$ von $s p\left(a^{\prime}\right), s p(b)$ erzeugt. $\operatorname{Im}$ zweiten Fall bestimmen $e=s p(a)$ und $e=s p(a) \circ t$ mit $z_{t}=\frac{1}{2} z_{t_{0}}$ die Möglichkeiten für $\bar{G}$.

Es gilt: $e \circ t_{0}^{i}=t_{0}^{i} \circ e, \mathrm{i}=1,-1 ; s p(a)^{2}=i d,(s p(a) \circ t)^{2}=t_{0}$.

Ein von $a, b, c$ berandeter Halbstreifen ist dann ein Fundamentalgebiet für $\bar{G}$ (s. Abb. 4).

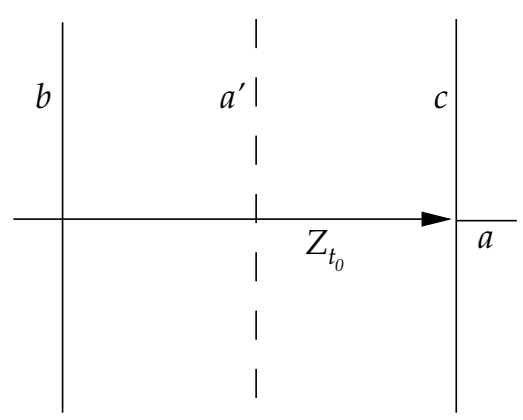

Abb. 4

Ist $G$ doppelt periodisch, so wählen wir zunächst wie im Beweis zu (3) minimale Erzeugende $t_{0}, t_{1}\left(\left|z_{t_{0}}\right| \leq\left|z_{t_{1}}\right|\right)$. Wir können $z_{t_{1}}$ so wählen, daß die Parallelkomponente zu $z_{t_{0}}$ in Richtung von $z_{t_{0}}$ zeigt und dem Betrage nach kleiner oder gleich $\frac{1}{2}\left|z_{t_{0}}\right|$ ist. Es sei $A$ die Anzahl der Perioden mit minimalem Betrag. Die drei folgenden Fallunterscheidungen umfassen sämtliche Möglichkeiten.

a) $A=2$

Dann haben nur die Perioden $z_{t_{0}},-z_{t_{0}}$ minimalen Betrag, also $\left|z_{t_{1}}\right|>\left|z_{t_{0}}\right|$.

Nach (5) muß die Menge der minimalen Perioden durch Spiegelung an einer möglichen Spiegelachse $a$ auf sich abgebildet werden. Für $a$ kommen damit nur die senkrecht und parallel zu $z_{t_{0}}$ verlaufenden Geraden in Frage. Die Möglichkeiten für $\bar{G}$ sind dann in (8) vollständig bestimmt. $\bar{G} \neq G$ gilt dann, wenn $z_{t_{0}}, z_{t_{1}}$ ein Rechteck aufspannen oder $z_{t_{1}}, z_{t_{0}}-z_{t_{1}}$ eine Raute. Zwei Gleitspiegelungen mit nicht parallelen Spiegelachsen können nach (6) in $\bar{G}$ nicht auftreten.

b) $A=4$

$z_{t_{1}}$ ist in diesem Fall eine minimale Periode; $\left|z_{t_{1}}\right|=\left|z_{t_{0}}\right|$. Verläuft $z_{t_{1}}$ nicht senkrecht zu $z_{t_{0}}$, so gibt es wiederum nur zwei mögliche Richtungen für die Spiegelachse $a$. Sie verlaufen parallel zu den Diagonalen der von $z_{t_{0}}, z_{t_{1}}$ aufgespannten Raute (s. Bemerkung unter a)). Wählt man in (8) $t=t_{1} \circ t_{0}, t^{\prime}=t_{1}$ bzw. $t=t_{1} \circ t_{0}^{-1}, t^{\prime}=$ $t_{0}^{-1}$, so erhält man sämtliche Möglichkeiten für $\bar{G}$. $\bar{G}$ wird dann durch $t_{0}, t_{1}, s p(a)$ erzeugt, wobei $a$ eine beliebige Gerade parallel zu einer Diagonalen ist. Verläuft $z_{t_{1}}$ senkrecht zu $z_{t_{0}}$, so gibt es neben den Richtungen der Diagonalen noch zwei weitere mögliche Richtungen für $a$, nämlich parallel zu $z_{t_{0}}$ bzw. $z_{t_{1}}$. Die zusätzlichen, $\bar{G}$ bestimmenden Erzeugenden $e$ erhält man dann nach (8).

c) $A=6$

In diesem speziellen Fall sind die Endpunkte der vom Nullpunkt aus abgetragenen minimalen Perioden die Eckpunkte eines regelmäßigen Sechsecks; $z_{t_{0}}, z_{t_{1}}, z_{t_{0}}-z_{t_{1}}$ 
bilden die Seiten eines gleichseitigen Dreiecks $\Delta$ (s. Abb. 5). Es gibt damit sechs mögliche Richtungen für $a$, die durch die Symmetrieachsen des Sechsecks oder durch die Seiten und Seitenhalbierenden von $\Delta$ bestimmt sind. Zu jeder dieser Richtungen gibt es Erzeugende von $G$ mit den in (8) angegebenen Voraussetzungen (Komponente von $z_{t^{\prime}}$ in Richtung $z_{t}$ ist $\frac{1}{2} z_{t}$ ). Damit bestimmen die Spiegelungen an Achsen, die parallel zu den oben angegebenen sechs Richtungen verlaufen, sämtliche Möglichkeiten für $\bar{G}$.

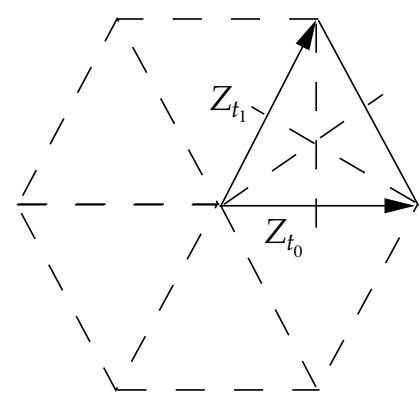

Abb. 5

Im Fall $A>6$ gibt es zwei minimale Perioden $z_{t_{0}}, z_{t_{1}} ;\left|z_{t_{0}}\right|=\left|z_{t_{1}}\right|$ mit $\left|z_{t_{1}}-z_{t_{0}}\right|<\left|z_{t_{0}}\right|$ im Widerspruch zur Wahl von $z_{t_{0}} ; G$ ist dann nicht diskret. Dieser Fall kann also nicht eintreten.

Zusammenfassend gilt:

Die doppelt periodische Gruppe $G(=U)$ kann genau dann durch eine zusätzliche Erzeugende $e$ zu einer Gruppe $\bar{G}$ erweitert werden, wenn es Erzeugende $t, t^{\prime}$ von $G$ gibt, deren Translationsvektoren $z_{t}, z_{t^{\prime}}$ zueinander senkrecht verlaufen oder vom gleichen Betrag sind.

Im ersten Fall sind die Möglichkeiten durch $e=s p(a)$; $a$ parallel zu einer der Symmetrieachsen des durch $z_{t}, z_{t^{\prime}}$ bestimmten Rechtecks $P$ bzw. durch die Gleitspiegelungen $e=s p(a) \circ t ; a$ parallel zu einer der Seiten von $P,\left|z_{t}\right|$ halbe Länge einer Seite von $P$; gegeben.

Verlaufen im zweiten Fall $z_{t}, z_{t^{\prime}}$ nicht senkrecht zueinander, so kann man die Erzeugenden $t, t^{\prime}$ so wählen, daß das von $z_{t}, z_{t^{\prime}}$ bestimmte Dreieck $\Delta$ spitzwinklig ist. Die Möglichkeiten sind dann durch $e=s p(a)$; a parallel oder senkrecht zu einer der Symmetrieachsen von $\Delta$; bestimmt.

\section{Die diskreten Gruppen mit Translationen und Drehungen}

Wir setzen nun im folgenden voraus, daß $G$ sowohl Drehungen als auch Translationen enthält. Die Untergruppe $U \subset G$ der Translationen ist diskret, nach (3) also einfach oder doppelt periodisch.

Wir untersuchen zunächst den Fall, daß $U$ einfach periodisch ist. 
Ist $t$ ein erzeugendes Element von $U$, so muß jede Drehung $j_{0} \in U$ die Richtung von $t$ auf eine parallele Richtung abbilden, also gilt ord $j_{0}=2$. Alle Drehungen in $G$ haben demnach einen Drehwinkel von $180^{\circ}$.

Schaltet man zwei derartige Drehungen hintereinander, so erhält man eine Translation in Richtung der Verbindungslinie der beiden Fixpunkte. Da $U$ einfach periodisch ist, müssen die Fixpunkte (Drehpunkte) aller Drehungen auf einer Geraden parallel zum Translationsvektor $z_{t}$ liegen.

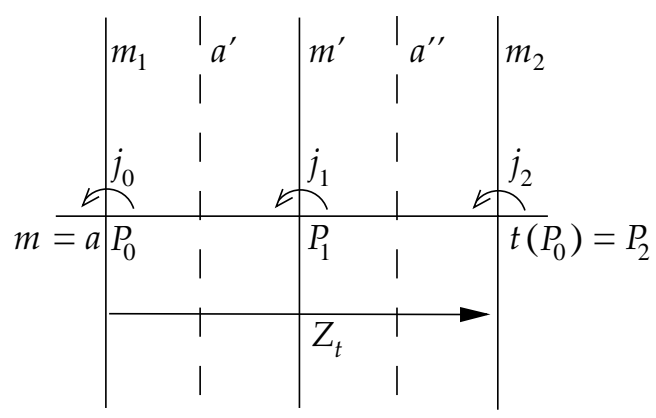

Abb. 6

Ist $P_{0}$ Fixpunkt der Drehung $j_{0}$, so ist der Mittelpunkt $P_{1}$ der Strecke $\overline{P_{0} t\left(P_{0}\right)}$ Fixpunkt der Drehung $j_{1}=t \circ j_{0}$.

Zwischen $P_{0}, P_{1}$ und $P_{1}, t\left(P_{0}\right)=P_{2}$ können keine weiteren Fixpunkte von Drehungen aus $G$ liegen, denn anderenfalls ist nach Obigem die Translation $t$ nicht minimal.

Es sei $G^{\prime}$ die von $j_{0}, j_{1}$ erzeugte Untergruppe von $G$.

Aus $j_{1} \circ j_{0}=t$ folgt, daß $t, j_{1} G^{\prime}$ erzeugen. Da jede Drehung aus $G$ die Form $t^{n} \circ j_{i} \circ t^{-n}$, $n \in \mathbb{Z}, i \in\{0,1\}$ hat, gilt $G^{\prime}=G$. Jedes Element hat dann wegen $j_{1}^{2}=i d ; t^{-1} \circ j_{1}=j_{1} \circ t$ eine eindeutige Darstellung $t^{m} \circ j_{1}^{i}$ mit $i \in\{0,1\}, m \in \mathbb{Z}$.

Ein Fundamentalgebiet $F$ ist dann ein von $m_{1}, m_{2}, m$ berandeter Halbstreifen (s. Abb. 6). Anhand der obigen Darstellung erkennt man sofort, daß die Bilder $g(F), g \in G$ die ganze Ebene lückenlos überdecken und sich nicht überlappen.

(9) Die diskreten Gruppen $G$ euklidischer Bewegungen mit einfach periodischen Untergruppen $U$ sind also die zyklischen Gruppen von Translationen und die oben beschriebenen unendlichen Dihedralgruppen.

Zur Bestimmung der Gruppen $\bar{G}$ kommen, da $G$ eine einfach periodische Untergruppe enthält, nur die im vorhergehenden Kapitel angegebenen Möglichkeiten in Frage. Die zusätzliche Erzeugende $e$ muß die Menge der Fixpunkte von Drehungen wieder auf sich abbilden. Dadurch ist die Wahl der Spiegelachsen $a$ eingeschränkt. Grundsätzlich sind damit $a=m, a=m^{\prime}, a=a^{\prime}$ (s. Abb. 6) möglich. 
Wegen

$$
j_{1} \circ s p\left(m^{\prime}\right)=s p(m) \circ s p\left(m^{\prime}\right) \circ s p\left(m^{\prime}\right)=s p(m)
$$

und

$$
j_{1} \circ s p\left(a^{\prime}\right)=s p(m) \circ s p\left(m^{\prime}\right) \circ s p\left(a^{\prime}\right)=s p(m) \circ \frac{1}{2} t
$$

sind durch $e=s p\left(a^{\prime}\right)$ und $e=s p\left(m^{\prime}\right)$ alle Möglichkeiten für $\bar{G}$ ausgeschöpft.

Es gilt: $s p\left(a^{\prime}\right) \circ j_{1}=t^{-1} \circ j_{1} \circ s p\left(a^{\prime}\right) ; s p\left(m^{\prime}\right) \circ j_{1}=j_{1} \circ s p\left(m^{\prime}\right)$. Jedes Element von $\bar{G}$ ist damit eindeutig darstellbar durch $t^{n} \circ j_{1}^{i} \circ e^{k}$ mit $n \in \mathbb{Z}, i, k \in\{0,1\}$. Fundamentalgebiete sind die durch $a^{\prime}, a^{\prime \prime}, m$ bzw. $m_{1}, m^{\prime}, m$ berandeten Halbstreifen (s. Abb. 6).

Wir behandeln nun den Fall, daß $U$ doppelt periodisch ist.

Wie im Beweis zu (3) wählen wir zunächst minimale Translationen $t_{0}, t_{1}$ mit den Perioden $z_{t_{0}}, z_{t_{1}} ;\left|z_{t_{0}}\right| \leq\left|z_{t_{1}}\right|$. Ist nun $P_{1}$ Fixpunkt einer Drehung aus $G$, so ist $t_{0}\left(P_{1}\right)=P_{2}$ ebenfalls Fixpunkt einer Drehung gleicher Ordnung $n$, die nach Voraussetzung ( $G$ diskret) endlich sein muß.

Das in Abbildung 1 dargestellte Dreieck $P_{1} P_{2} Q$ ist dann gleichschenklig, der Betrag des Translationsvektors $\overrightarrow{Q_{1}}$ ist nach Konstruktion höchstens doppelt so groß wie der Betrag von $\overrightarrow{Q Q_{2}}$.

Für $n \geq 7$ gilt: $\alpha \leq \frac{\pi}{7} ;\left|\overrightarrow{Q Q_{1}}\right| \leq 2 \cdot\left|\overrightarrow{Q Q_{2}}\right| \leq 2 \cdot 2 \cdot \tan \alpha \cdot \frac{1}{2}\left|\overrightarrow{P_{1} P_{2}}\right|=$

$$
2 \cdot \tan \alpha \cdot\left|\overrightarrow{P_{1} P_{2}}\right| \leq 2 \cdot 0,49 \cdot\left|\overrightarrow{P_{1} P_{2}}\right|<\left|\overrightarrow{P_{1} P_{2}}\right|=\left|z_{t_{0}}\right|
$$

Das widerspricht der Voraussetzung über $z_{t_{0}}$. Es folgt also:

(10) Ist $U \subset G$ doppelt periodisch, so haben die Drehungen aus $G$ eine Ordnung, die höchstens gleich 6 ist.

In der nachfolgenden Tabelle sind anhand der Abbildung 1 sämtliche Möglichkeiten dargestellt. Die Ordnungen der Drehungen $j_{1}, j_{2}, j_{3}$ mit den Fixpunkten $P_{1}$ bzw. $P_{2}$ bzw. $Q=P_{3}$ seien $n_{1}$ bzw. $n_{2}$ bzw. $n_{3}$. $n_{3}$ berechnet sich aus $n_{3} 2 \gamma=k 360 \Leftrightarrow n_{3} \gamma=$ $k 180 ; n_{3}, k \in \mathbb{N} ; n_{3}, k$ minimal.

\begin{tabular}{rrrrrrc}
$n_{1}$ & $n_{2}$ & $\alpha\left[^{\circ}\right]$ & $\beta\left[^{\circ}\right]$ & $\gamma\left[^{\circ}\right]$ & $n_{3}$ & $\begin{array}{c}\text { möglich (m) bzw. } \\
\text { nicht möglich }(\mathrm{nm})\end{array}$ \\
\hline 6 & 6 & 30 & 30 & 120 & 3 & $\mathrm{~m}$ \\
6 & 5 & 30 & 36 & 114 & 30 & $\mathrm{~nm}$ \\
6 & 4 & 30 & 45 & 105 & 12 & $\mathrm{~nm}$ \\
6 & 3 & 30 & 60 & 90 & 2 & $\mathrm{~m}$ \\
6 & 2 & 30 & 90 & 60 & 3 & $\mathrm{~m}$ \\
5 & 5 & 36 & 36 & 108 & 5 & $\mathrm{~m}$ \\
5 & 4 & 36 & 45 & 99 & 20 & $\mathrm{~nm}$ \\
5 & 3 & 36 & 60 & 84 & 15 & $\mathrm{~nm}$ \\
5 & 2 & 36 & 90 & 54 & 10 & $\mathrm{~nm}$ \\
4 & 4 & 45 & 45 & 90 & 2 & $\mathrm{~m}$ \\
4 & 3 & 45 & 60 & 75 & 12 & $\mathrm{~nm}$ \\
4 & 2 & 45 & 90 & 45 & 4 & $\mathrm{~m}$ \\
3 & 3 & 60 & 60 & 60 & 3 & $\mathrm{~m}$ \\
3 & 2 & 60 & 90 & 30 & 6 & $\mathrm{~m}$
\end{tabular}


Nach der obigen Tabelle kommen wegen (10) damit nur fünf Möglichkeiten für $G$ in Frage. Die Ordnungen der Drehungen von $G(\operatorname{ord}(j))$ können sein 1.) sämtlich 2, 2.) sämtlich 3, 3.) 4 oder 2, 4.) 6 oder 3 oder 2, 5.) sämtlich 5.

Der 5. Fall ist leicht auszuschließen. In diesem Fall ist (trotz gleicher Ordnung) das Basisdreieck $P_{1} Q P_{2}$ nicht gleichseitig. Es kann demnach entgegen der Voraussetzung über $G$ keinen positiven minimalen Abstand zweier Fixpunkte (Drehpunkte) geben; $G$ ist unter diesen Voraussetzungen nicht diskret.

Die verbleibenden Fälle 1. bis 4. werden nun im einzelnen näher untersucht. Die Drehungen werden weiterhin grundsätzlich mit $j$, Translationen mit $t$ bezeichnet; $j_{i}$ ist die Drehung mit minimalem positivem Drehwinkel um den Punkt $P_{i}$. Die hergeleiteten notwendigen Eigenschaften für $G$ und $\bar{G}$ sind auch hinreichend, was aus der Untersuchung unmittelbar ersichtlich ist. Wir werden darauf nicht in jedem Fall wieder hinweisen.

1. $\operatorname{ord}(j)=2$ für alle $j \in G$

Wir können nach eventueller Konjugation davon ausgehen, daß $P_{0}=0$ Fixpunkt einer Drehung ist. Dann sind auch die weiteren Eckpunkte $P_{2}, P_{4}, P_{6}$ des von den Vektoren $z_{t_{0}}$ und $z_{t_{1}}$ aufgespannten abgeschlossenen Parallelogramms $P$ Fixpunkte von Drehungen. Der Beweis zu (9) (s. Abb. 6) zeigt, daß die in der Abb. 7 eingetragenen Punkte $P_{i}, i=$ $1, \ldots, 8$, sämtlich Fixpunkte von Drehungen sind.

Man erkennt leicht, da $ß$ in $P$ keine weiteren Fixpunkte liegen. Die Bilder $t(P), t \in U$ überdecken lückenlos ganz $E$. Die Fixpunkte von Drehungen aus $G$ sind demnach Bilder der in $P$ liegenden Fixpunkte unter $U$; sämtliche Drehungen aus $G$ somit konjugiert unter $U$ zu den Drehungen mit Fixpunkten in $P$.

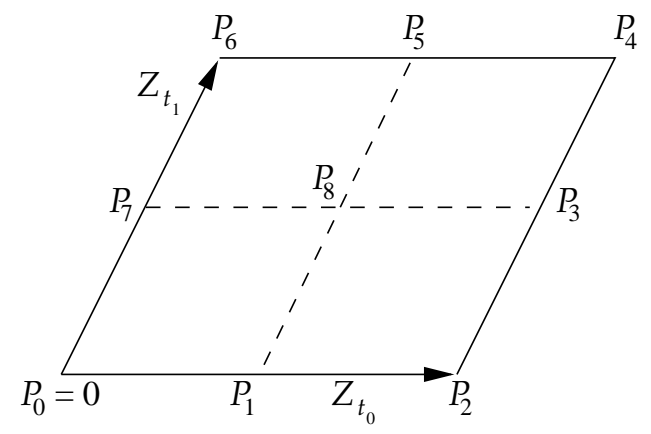

Abb. 7

Wegen $j_{1}=j_{8} \circ t_{1} ; j_{0}=j_{1} \circ t_{0} ; j_{7}=j_{8} \circ t_{0}$ erzeugen $j_{8}, t_{0}, t_{1}$ ganz $G$.

Mit $j_{i}=j_{i}^{-1}, i=1, \ldots, 8$ gilt $j_{8} \circ t_{1}^{m}=t_{1}^{-m} \circ j_{8} ; j_{8} \circ t_{0}^{m}=t_{0}^{-m} \circ j_{8}, m=-1,1$.

Damit läßt sich jede Transformation aus $G$ in der Form $t \circ j_{8}^{k}, k \in\{0,1\}$ mit $t \in U$ eindeutig darstellen. Hieraus wiederum erkennt man, daß das Parallelogramm mit den Eckpunkten $P_{0}, P_{2}, P_{3}, P_{7}$ ein Fundamentalgebiet $F$ von $G$ ist. Die Bilder $g(F), g \in G$, sind dann Translationen von $F(k=0)$ oder Translationen von $j_{8}(F)$ (Parallelogramm $\left.P_{7} P_{3} P_{4} P_{6} ; k=1\right)$, überdecken somit die Ebene $E$ lückenlos. 
Bei der Bestimmung der Gruppen $\bar{G}$ kommen nur die im vorhergehenden Kapitel angegebenen Erzeugenden $e$ für den doppelt periodischen Fall in Frage. Die zugehörigen Spiegelachsen $a$ sind hier durch die Lage der Fixpunkte festgelegt. Jede Erzeugende $e$ muß die Menge der Fixpunkte wieder auf sich abbilden.

Wie dort unterscheiden wir drei Fälle.

(a) $A=2$

Spannen $z_{t_{0}}, z_{t_{1}}$ ein Rechteck auf, so können mögliche Spiegelachsen $a$ nur in Richtung der Seiten dieses Rechtecks verlaufen. Da zudem die Erzeugende $e$ die Fixpunktmenge auf sich abbildet, kann grundsätzlich $a$ nur so verlaufen wie eine der in der Abbildung 8 angegebenen Achsen $a_{1}, a_{2}, a_{3}, a_{4}$. Wegen $j_{8} \circ s p\left(a_{1}\right)=s p\left(a_{4}\right) \circ$ $\frac{1}{2} t_{0} ; j_{8} \circ s p\left(a_{1}\right) \circ \frac{1}{2} t_{1}=s p\left(a_{3}\right) \circ \frac{1}{2} t_{0} ; j_{8} \circ s p\left(a_{2}\right)=s p\left(a_{4}\right) ; j_{8} \circ s p\left(a_{3}\right)=s p\left(a_{2}\right) \circ \frac{1}{2} t_{1}$ sind alle Möglichkeiten durch $a=a_{i}, i=1,2,3 ; e=s p\left(a_{i}\right)$ und $e=s p\left(a_{1}\right) \circ \frac{1}{2} t_{1}$ erfaßt.

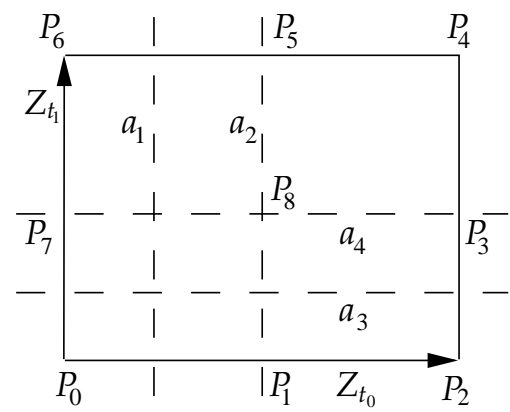

Abb. 8

Schaltet man von diesen Erzeugenden zwei verschiedene hintereinander, so erhält man eine Translation oder eine Drehung, die nicht in $G$ liegt. Jede Gruppe $\bar{G}$ enthält demnach nur eine derartige zusätzliche Erzeugende. Aus $s p\left(a_{1}\right) \circ j_{8}=$ $j_{7} \circ s p\left(a_{1}\right) ; s p\left(a_{2}\right) \circ j_{8}=j_{8} \circ s p\left(a_{2}\right) ; s p\left(a_{3}\right) \circ j_{8}=j_{1} \circ s p\left(a_{3}\right) ; s p\left(a_{1}\right) \circ \frac{1}{2} t_{1} \circ j_{8}=$ $j_{6} \circ s p\left(a_{1}\right) \circ \frac{1}{2} t_{1}$ und der Tatsache, daß $j_{8}, t_{0}, t_{1}$ Erzeugende von $G$ sind, folgt die eindeutige Darstellung einer Transformation aus $\bar{G}$ in der Form $t \circ j_{8}^{i} \circ e^{k}$ mit $t \in U, i, k \in\{0,1\}$.

Ist das von $z_{t_{0}}, z_{t_{1}}$ aufgespannte Parallelogramm kein Rechteck, so gibt es nur zusätzliche Erzeugende $e$, wenn $\left|z_{t_{1}}-z_{t_{0}}\right|=\left|z_{t_{1}}\right|$ gilt. Mögliche Spiegelachsen $a$ müssen parallel oder senkrecht zu $z_{t_{0}}$ verlaufen. Hier jedoch trifft jede mögliche Spiegelachse einen Fixpunkt ( $a=a_{1}, a_{2}$ verlaufen durch Fixpunkte). Die Spiegelungen an Geraden $a$, die senkrecht oder parallel zu $z_{t_{0}}$ verlaufen und zudem einen Fixpunkt treffen, ergeben sämtliche Möglichkeiten für $e$. Sind $a, a^{\prime} ; a \perp a^{\prime}$; zwei derartige Achsen, die sich in einem gemeinsamen Fixpunkt der Drehung $j \in G$ treffen, so gilt $s p\left(a^{\prime}\right) \circ j=s p(a)$. Die Spiegelungen an Geraden parallel oder senkrecht zu $z_{t_{0}}$, die durch einen Fixpunkt verlaufen und sich paarweise nicht in einem Fixpunkt einer Drehung aus $G$ schneiden, umfassen dann sämtliche Möglichkeiten für $e$. 
(b) $A=4$

Ist die von $z_{t_{0}}, z_{t_{1}}$ aufgespannte Raute ein Quadrat, so sind die unter (a) im Zusammenhang mit dem Rechteck bestimmten Erzeugenden $e$ auch hier zusätzliche Erzeugende. Hinzu kommen noch die Spiegelungen an Achsen, die parallel zu einer der Diagonalen des Quadrats durch einen Fixpunkt verlaufen. Treffen sich zwei dieser Achsen senkrecht in einem Fixpunkt der Drehung $j \in G$, so ist mit der Spiegelung an einer der Achsen auch die Spiegelung an der anderen Achse in $\bar{G}$ enthalten (s.o. $s p\left(a^{\prime}\right) \circ j=s p(a)$ ).

Ist die von $z_{t_{0}}, z_{t_{1}}$ aufgespannte Raute kein Quadrat, so bestimmen die Spiegelungen an Achsen parallel zu einer der Diagonalen durch einen Fixpunkt schon sämtliche Möglichkeiten für $e$.

(c) $A=6$

Hier verlaufen die möglichen Achsen durch einen Fixpunkt einer Drehung $j \in$ $G$ parallel zu einer der sechs möglichen Richtungen. Treffen sich zwei derartige Achsen senkrecht in einem Fixpunkt, so ist mit der Spiegelung an einer der Achsen auch die Spiegelung an der anderen Achse in $\bar{G}$ enthalten.

2. $\operatorname{ord}(j)=3$ für alle Drehungen $j \in G$

Da $G$ diskret ist, können die endlichen Fixpunkte der Drehungen sich in keinem Punkt häufen. Es gibt somit zwei Drehungen $j_{1}, j_{2}$, deren Fixpunkte $P_{1}, P_{2}$ minimalen Abstand haben.

Die Konstruktion nach Abbildung 1 liefert ein gleichseitiges Dreieck $\Delta=P_{1} P_{2} P_{3}\left(P_{3}=\right.$ $Q)$, in dessem Innern sowie auf dem Rande keine weiteren Fixpunkte von Elementen aus $G$ liegen können, da sonst der Abstand von $P_{1}$ zu $P_{2}$ nicht minimal ist. Das gleiche gilt für das Dreieck $\Delta^{\prime}=P_{1} P_{2} P_{7}$ (s. Abb. 9). Zudem erkennt man anhand der Konstruktion, daß $G$ eine doppelt periodische Untergruppe $U$ enthält. Es sei $G^{\prime} \subseteq G$ die von $j_{1}, j_{2}$ erzeugte Untergruppe; $G^{\prime}=<j_{1}, j_{2}>$. Dann gilt (s. Abb. 9):

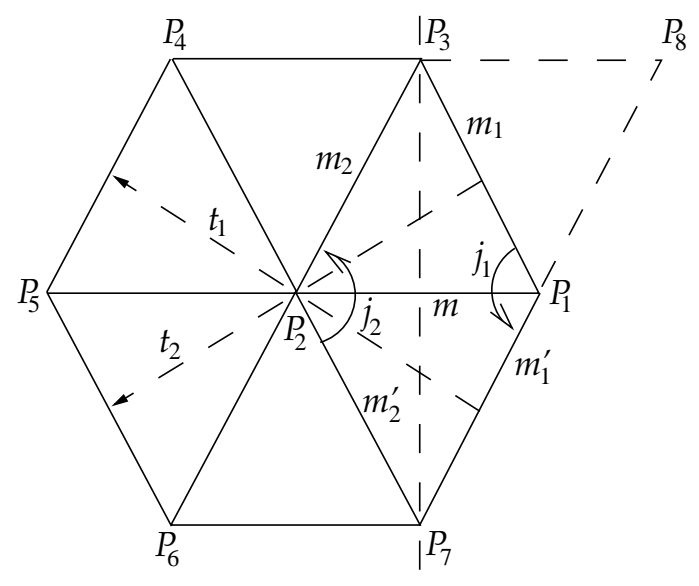

Abb. 9 
$j_{1}^{2}=j_{1}^{-1}=s p(m) \circ s p\left(m_{1}^{\prime}\right) ;$

$j_{2}=s p\left(m_{2}\right) \circ s p(m)$

$j_{2} \circ j_{1}^{-1}=s p\left(m_{2}\right) \circ s p\left(m_{1}^{\prime}\right)=t_{1}$;

$j_{2}^{-1} \circ j_{1}=s p\left(m_{2}^{\prime}\right) \circ s p(m) \circ s p(m) \circ s p\left(m_{1}\right)=s p\left(m_{2}^{\prime}\right) \circ s p\left(m_{1}\right)=t_{2}$.

$t_{1}, t_{2}$ sind Translationen aus $G^{\prime}$.

Ihre Vektoren $z_{t_{1}}, z_{t_{2}}$ haben einen Betrag, der doppelt so groß wie der Betrag der Höhe im Dreieck $P_{1} P_{3} P_{2}$ ist. $G^{\prime}$ wird also von $t_{2}, j_{2}$ erzeugt. Aus den obigen Gleichungen folgt direkt: $j_{2} \circ t_{2}=j_{1}=t_{1}^{-1} \circ j_{2} ; j_{2} \circ t_{2}^{-1}=j_{2} \circ j_{1}^{-1} \circ j_{2}=t_{1} \circ j_{2} ; j_{2} \circ t_{1}=t_{2} \circ t_{1}^{-1} \circ j_{2}$; $j_{2} \circ t_{1}^{-1}=t_{1} \circ t_{2}^{-1} \circ j_{2}$. Hieraus wiederum folgt, daß jede Transformation aus $G^{\prime}$ sich in der Form $t \circ j_{2}^{k}$ mit $k \in\{0,1,2\}, t \in U$ ( $U-$ von $t_{1}, t_{2}$ erzeugte Untergruppe von $\left.G^{\prime}\right)$, darstellen läßt. Gehen wir vom Parallelogramm $F$ mit den Eckpunkten $P_{1}, P_{3}, P_{2}, P_{7}$ der Abbildung 9 aus, so bilden $j_{2}^{0}(F)=F, j_{2}(F), j_{2}^{2}(F)$ zusammen ein regelmäßiges Sechseck. Die Translationen $t \in G^{\prime}$ bilden dieses Sechseck wieder auf Sechsecke ab, die insgesamt $E$ lückenlos überdecken. Jeweils zwei dieser Bilder haben höchstens gemeinsame Randpunkte. Die Fixpunkte sämtlicher Drehungen aus $G$ sind demnach die Bildpunkte von $P_{1}, P_{2}, P_{3}, P_{7}$ unter $G^{\prime}$. Jedes Element $g$ aus $G$ bildet das Dreieck $\Delta$ auf ein kongruentes Dreieck ab, dessen Eckpunkte wiederum Fixpunkte von Drehungen sind. Es gibt also ein $g^{\prime} \in G^{\prime}$ mit $g^{\prime} \circ g(\Delta)=\Delta$ oder $g^{\prime} \circ g(\Delta)=\Delta^{\prime}$.

Da $G^{\prime}$ sämtliche Drehungen aus $G$ enthält und keine dieser Drehungen $\Delta$ auf sich oder auf $\Delta^{\prime}$ abbildet - es gibt zudem keine Translation, die $\Delta$ in $\Delta^{\prime}$ überführt -, folgt $g^{\prime} \circ g=i d, g \in G^{\prime}$, also $G^{\prime}=G$. Die Struktur von $G$ ist somit vollständig überschaubar.

Es sei nun $e$ eine ungerade Mehrfachspiegelung, also eine Gleitspiegelung. Da mit $P$ auch $e(P)$ Fixpunkt einer Drehung aus $G$ ist, bildet $e$ das Dreieck $\Delta$ auf ein dazu kongruentes Dreieck ab, das zugleich das Bild von $\Delta$ oder von $\Delta^{\prime}$ unter $G$ ist. Es gibt also ein $g \in G$ mit $g \circ e(\Delta)=\Delta$ oder $g \circ e(\Delta)=\Delta^{\prime}$.

Wir untersuchen zunächst den Fall $g \circ e(\Delta)=\Delta$.

Wegen $g \circ e=t \circ s p=s p \circ t ;(g \circ e)^{2}=t \circ s p \circ s p \circ t=t^{2}$ und $(g \circ e)^{2}(\Delta)=\Delta$, verschwindet der Translationsanteil der Gleitspiegelung $g \circ e$, also ist $g \circ e$ eine einfache Spiegelung $s$, die $\Delta$ auf sich abbildet. Die Spiegelachse von $s$ kann damit nur eine Seitenhalbierende von $\Delta$ sein. Wir nehmen an, es sei die Seitenhalbierende durch $P_{3}$ (s. Abb. 9).

Wegen

$$
\begin{array}{cl}
s \circ j_{1}=j_{2}^{-1} \circ s & \text { (beide Transformationen bilden } P_{1} \text { auf } P_{2} \\
& \text { und } P_{3} \text { auf } P_{7} \text { ab); } \\
s \circ j_{2}=j_{1}^{-1} \circ s & \left(P_{2} \longrightarrow P_{1} ; P_{7} \longrightarrow P_{3}\right) ; \\
j_{i}^{2}=j_{i}^{-1}, & i=1,2,
\end{array}
$$

läßt sich jede Transformation aus $\bar{G}$ in der Form $g \circ s^{i}$ mit $g \in G, i \in\{0,1\}$ und damit durch $t \circ j_{2}^{k} \circ s^{i} ; t \in U, k \in\{0,1,2\}, i \in\{0,1\}$ eindeutig darstellen. Aus dieser Darstellung erkennen wir, daß Dreieck $P_{7} P_{3} P_{2}$ ein Fundamentaldreieck von $\bar{G}$ ist. 
Ist $g \circ e(\Delta)=\Delta^{\prime}$ und $m$ die Gerade $P_{1} P_{2}$, so gilt $s p(m) \circ g \circ e(\Delta)=\Delta . s p(m) \circ g \circ e=j$ ist dann entweder eine Drehung der Ordnung 3 um den Schwerpunkt von $\Delta$ oder die Identität.

Im 1. Fall ist $g \circ e=s p(m) \circ j$ eine Gleitspiegelung mit nicht verschwindendem Translationsanteil und damit $(g \circ e)^{2}=(s p(m) \circ j)^{2}$ eine Translation, die entweder $P_{2}$ auf $P_{7}$ oder $P_{2}$ auf $P_{6}$ abbildet. In beiden Fällen überlappen sich die Bilder des Sechsecks $\operatorname{Se}\left(P_{1} P_{3} P_{4} P_{5} P_{6} P_{7}\right)$ mit dem Sechseck selbst. Da jede Translation aus $G S e$ auf ein Sechseck abbildet, das keine gemeinsamen inneren Punkte mit $S e$ hat (s. Darstellung der Elemente aus $G)$, kann dann entgegen der Annahme $(g \circ e)^{2}$ nicht in $G$ enthalten sein; $j$ ist also keine Drehung. Damit folgt $s p(m) \circ g \circ e=i d ; g \circ e=s p(m)$. Wegen $s p(m) \circ j_{1}$ $=s p\left(m_{1}\right)=j_{1}^{-1} \circ s p(m) ; s p(m) \circ j_{2}=s p\left(m_{2}^{\prime}\right)=j_{2}^{-1} \circ s p(m) ; j_{i}^{2}=j_{i}^{-1}, i=1,2$, läßt sich jedes Element aus $\bar{G}$ in der Form $t \circ j_{2}^{k} \circ s p(m)^{i}, t \in U, k \in\{0,1,2\}, i \in\{0,1\}$ eindeutig darstellen. Wir erkennen aus der obigen Darstellung, daß $\Delta$ in diesem Fall ein Fundamentaldreieck ist. Die Bilder $\mathrm{g}(\Delta)$ mit $g \in \bar{G}$ parkettieren die Ebene $E$. Aus den obigen Gleichungen folgt zudem, daß $s p(m), s p\left(m_{1}\right), s p\left(m_{2}\right)$ ganz $\bar{G}$ erzeugen. Alle Dreiecke der Parkettierung erhält man durch fortgesetzte Spiegelung an den Dreieckseiten $m, m_{1}, m_{2}$. Damit sind alle Möglichkeiten der Erweiterung von $G$ zu $\bar{G}$ erschöpft. Die zusätzlichen Erzeugenden sind grundsätzlich die Spiegelungen an den Diagonalen einer Fundamentalraute (etwa $P_{1} P_{3} P_{2} P_{7}$ ) von $G$. Die Diagonalen zerlegen eine derartige Raute jeweils in Dreiecke $\left(P_{1} P_{2} P_{3}\right.$ bzw. $\left.P_{3} P_{2} P_{7}\right)$, die Fundamentalgebiete von $\bar{G}$ sind.

3. $\operatorname{ord}(j)=4$ oder $\operatorname{ord}(j)=2$ für $j \in G$. Wir folgen dem im vorhergehenden Fall gekennzeichneten Lösungsweg zur Bestimmung von $G, \bar{G}$. Die Drehungen $j_{1}, j_{2}$ mit minimalem Abstand der Fixpunkte haben nach Abbildung 1 verschiedene Ordnungen. Es gilt (s. Abb. 10): $j_{2}^{2} \circ j_{1}=s p\left(m^{\prime}\right) \circ s p(m) \circ s p(m) \circ s p\left(m_{1}\right)=s p\left(m^{\prime}\right) \circ s p\left(m_{1}\right)=t_{1}$ $j_{2} \circ j_{1} \circ j_{2}=j_{3}^{-1} \circ j_{2}=s p\left(m^{\prime \prime}\right) \circ s p\left(m_{2}\right) \circ s p\left(m_{2}\right) \circ s p(m)=s p\left(m^{\prime \prime}\right) \circ s p(m)=t_{2}$

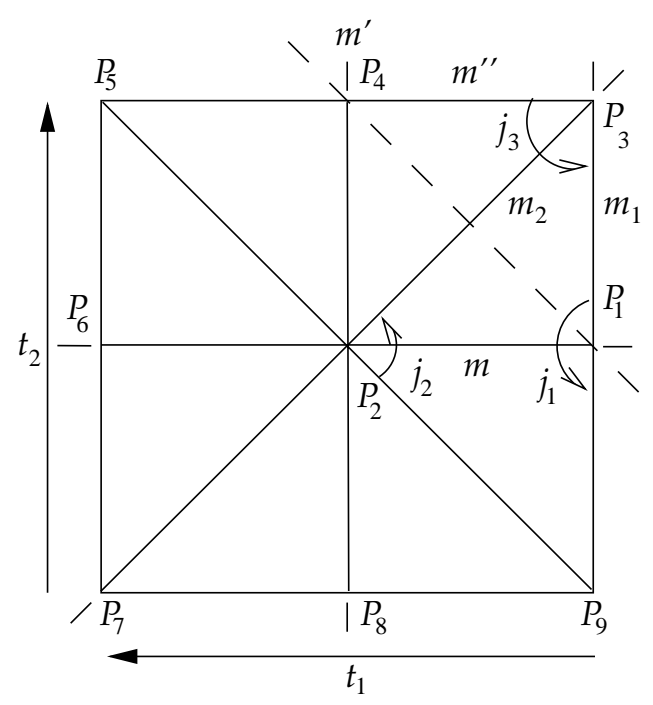

Abb. 10 
Wie im vorhergehenden Fall schließt man, daß sich jedes Element aus $G^{\prime}=<j_{1}, j_{2}>$ eindeutig durch $t \circ j_{2}^{i}, t \in U, i \in\{0,1,2,3\}$ darstellen läßt.

Hieraus erkennt man, daß das Dreieck $P_{3} P_{2} P_{9}$ ein Fundamentalgebiet $F$ ist und die Bilder $g(F)$ mit $g \in G^{\prime}$ die Ebene $E$ parkettieren. Da es keine Drehung in $G$ gibt, die $\Delta\left(P_{1} P_{3} P_{2}\right)$ auf sich oder auf $\Delta^{\prime}\left(P_{1} P_{2} P_{9}\right)$ abbildet, schließt man wie im vorhergehenden Fall $G^{\prime}=G$.

Nur die Spiegelung s an der Seitenhalbierenden von $\Delta$ durch $P_{1}$ bildet $\Delta$ auf sich ab. Ähnlich wie im vorhergehenden Fall schließt man, daß $\bar{G}$ entweder durch $s, j_{1}, j_{2}$ (Fundamentalgebiet $P_{1} P_{3} P_{4}$ ) oder durch $s p(m), j_{1}, j_{2}$ (Fundamentalgebiet $\Delta$ ) erzeugt wird. Im zweiten Fall gilt $s p(m), s p\left(m_{1}\right), s p\left(m_{2}\right) \in \bar{G}$; jede Transformation erhält man durch mehrfache Spiegelung an den Seiten des Fundamentaldreiecks $\Delta$.

4. $\operatorname{ord}(j)=6$ oder $\operatorname{ord}(j)=3$ oder $\operatorname{ord}(j)=2$ für $j \in G$

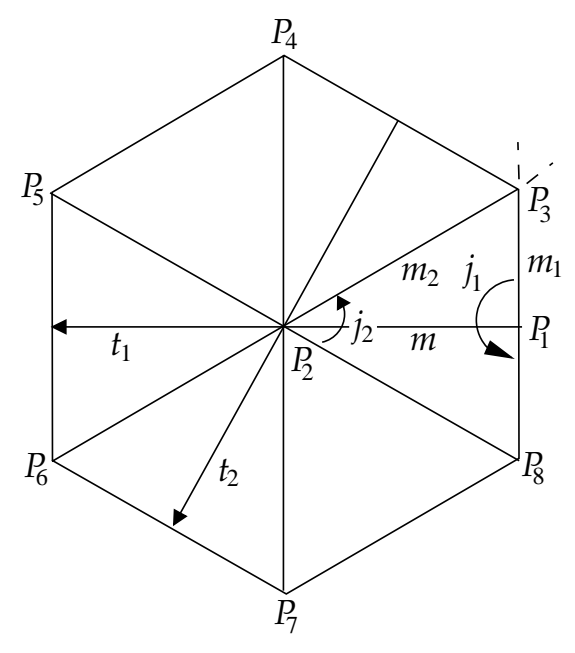

Abb. 11

Hier haben die Fixpunkte $P_{1}, P_{3}$ minimalen Abstand. Es gilt (s. Abb. 11):

$$
\begin{aligned}
j_{2}^{3} \circ j_{1} & =t_{1} & & \left(P_{3} \rightarrow P_{5} ; P_{8} \rightarrow P_{6}\right) ; \\
j_{2} \circ t_{1} \circ j_{2}^{-1} & =j_{2}^{4} \circ j_{1} \circ j_{2}^{-1}=t_{2} & & \left(P_{3} \rightarrow P_{7} ; P 4 \rightarrow P_{6}\right) ;
\end{aligned}
$$

Wiederum erhält man eine Darstellung $t \circ j_{2}^{i}, i \in\{0,1,2,3,4,5\}, t \in U$, für alle Elemente $g \in G^{\prime}=<j_{2}, t_{1}>$. Wie in den vorhergehenden Fällen folgt $G^{\prime}=G$ mit dem Fundamentalgebiet $P_{2} P_{8} P_{3}$; es gibt keine Drehung in $G$, die $\Delta\left(P_{1} P_{3} P_{2}\right)$ auf sich oder $\Delta^{\prime}\left(P_{1} P_{2} P_{8}\right)$ abbildet. Die Gruppe $\bar{G}$ mit dem Fundamentalgebiet $\Delta$ ist hier eindeutig, da $\Delta$ keine Symmetrieachse hat. Sie wird von den Spiegelungen $s p(m), s p\left(m_{1}\right), s p\left(m_{2}\right)$ erzeugt. 


\section{Übersicht}

$$
\begin{aligned}
& \left\langle s_{1}, \ldots, s_{n}\right\rangle-\text { die von den Elementen } s_{1}, \ldots, s_{n} \text { erzeugte Gruppe; } \\
& j_{(n)}^{\prime}, j_{(n)} \text { - Drehungen der Ordnung } \mathrm{n} ; \\
& t_{0}, t_{1}-\text { minimale Erzeugende der doppelt periodischen Untergruppe } U \text { mit } \\
& \quad\left|z_{t_{0}}\right| \leq\left|z_{t_{1}}\right| \leq\left|z_{t}\right| \text { für alle } t \in U ; 60^{\circ} \leq \Varangle\left(z_{t_{0}}, z_{t_{1}}\right) \text { oder } \Varangle\left(z_{t_{1}}, z_{t_{0}}\right) \leq 90^{\circ} ; \\
& s p(a) \circ t-\text { Gleitspiegelung an der Achse } a \text { mit } z_{t}=\frac{1}{2} z_{t_{0}} \text { oder } z_{t}=\frac{1}{2} z_{t_{1}} ; \\
& \bar{\Delta}, P \text { - ein durch } z_{t_{0}}, z_{t_{1}} \text { bestimmtes Dreieck bzw. aufgespanntes Parallelogramm; } \\
& \bar{G}-\text { wird von } G \text { und } e \text { erzeugt. }
\end{aligned}
$$

\begin{tabular}{|c|c|c|}
\hline G & e & \\
\hline$\{i d\}$ & $s p(a)$ & $a$ - beliebige Gerade \\
\hline $\begin{array}{l}\left.j_{(n)}\right\rangle \text { endlich zy- } \\
\text { klisch }\end{array}$ & $s p(a)$ & $\begin{array}{l}a \text { - beliebige Gerade durch das Drehzentrum von } \\
j_{(n)}\end{array}$ \\
\hline $\begin{array}{l}\left\langle t_{0}\right\rangle(=\mathrm{U}) \text { unendlich } \\
\text { zyklisch (einfach } \\
\text { periodisch) }\end{array}$ & $\begin{array}{l}s p(a) \\
s p(a) \circ t\end{array}$ & $a \perp z_{t_{0}} ;$ oder $a \| z_{t_{0}}$ \\
\hline \multicolumn{3}{|l|}{$\begin{array}{l}\left\langle t_{0}, t_{1}\right\rangle(=\mathrm{U}) \text { doppelt } \\
\text { periodisch }\end{array}$} \\
\hline a) $z_{t_{1}} \perp z_{t_{0}}$ & $s p(a)$ & $\begin{array}{l}a-\text { parallel zu einer der Symmetrieachsen von } P \text {; } \\
\text { oder }\end{array}$ \\
\hline & $s p(a) \circ t$ & $a$ - parallel zu einer Seite von $P$ \\
\hline $\begin{array}{l}\beta) z_{t_{1}} \text { nicht senk- } \\
\text { recht zu } z_{t_{0}} \text { und } \bar{\Delta} \\
\text { gleichschenklig }\end{array}$ & $s p(a)$ & $\begin{array}{l}a-\text { parallel oder senkrecht zu einer Symmetrieachse } \\
\text { von } \bar{\Delta}\end{array}$ \\
\hline $\begin{array}{l}\gamma) z_{t_{1}} \text { nicht senk- } \\
\text { recht zu } z_{t_{0}} \text { und } \bar{\Delta} \\
\text { nicht gleichschenk- } \\
\text { lig }\end{array}$ & & es gibt keine Möglichkeit für $e$ \\
\hline $\begin{array}{l}\left\langle j_{(2)}, t_{0}\right\rangle U \text { ist ein- } \\
\text { fach periodisch }\end{array}$ & $s p(a)$ & $\begin{array}{l}a \perp z_{t_{0}} \text {; a verläuft durch den Fixpunkt einer Dre- } \\
\text { hung aus } G \text { oder durch den Mittelpunkt einer Strek- } \\
\text { ke mit der Länge } \frac{1}{2}\left|z_{t_{0}}\right| \text {, deren Endpunkte benach- } \\
\text { barte Fixpunkte aus } G \text { sind. }\end{array}$ \\
\hline $\begin{array}{l}\left\langle j_{(2)}, t_{0}, t_{1}\right\rangle U \text { ist } \\
\text { doppelt periodisch }\end{array}$ & & $\begin{array}{l}\text { s. Fälle } \alpha), \beta), \gamma) \text { mit der Einschränkung, daß durch } \\
\text { die Spiegelung an } a \text { die Fixpunktmenge auf sich } \\
\text { abgebildet wird. }\end{array}$ \\
\hline$\left\langle j_{(3)}^{\prime}, j_{(3)}\right\rangle$ & $s p(a)$ & $\begin{array}{l}a \text { - Seitenhalbierende oder Seite eines gleichseiti- } \\
\text { gen Basisdreiecks, dessen Seitenlänge gleich dem } \\
\text { minimalen Abstand zweier Fixpunkte ist. }\end{array}$ \\
\hline
\end{tabular}




\begin{tabular}{c|cl}
$\mathrm{G}$ & $e$ & \\
\hline$\left\langle j_{(2)}, j_{(4)}\right\rangle$ & $s p(a) ;$ & $\begin{array}{l}a-\text { Symmetrieachse oder Seite eines gleichschenk- } \\
\text { ligen, rechtwinkligen Basisdreiecks, dessen Kathe- } \\
\text { tenlänge gleich dem minimalen Abstand zweier Fix- } \\
\text { punkte ist. }\end{array}$ \\
\hline$\left\langle j_{(2)}, j_{(3)}\right\rangle$ & $s p(a) ;$ & $\begin{array}{l}a-\text { Seite eines rechtwinkligen Basisdreiecks, bei } \\
\text { dem eine Kathetenlänge gleich dem minimalen Ab- } \\
\text { stand zweier Fixpunkte ist. } \bar{G} \text { ist eindeutig. }\end{array}$
\end{tabular}

\section{Literatur}

[1] L.K. Ford. Automorphic Functions. Chelsea, New York, 1951.

[2] I. Kra. Automorphic Forms and Kleinian Groups. W.A. Benjamin, Inc., 1972.

[3] J. Lehner. Discontinuous Groups and Automorphic Functions. American Mathematical Society, Providence, Rhode Island, 1964.

[4] B. Maskit. Kleinian Groups. Springer Verlag, Berlin Heidelberg, 1988.

Walter Wolking

Holdorferstr. 1

D-49401 Damme 